\title{
Ethnologies
}

\section{La représentation des immigrants dans l'espace muséal et patrimonial de Québec}

\section{Nada Guzin Lukić}

Volume 27, numéro 1, 2005

Appartenances

Belonging

URI : https://id.erudit.org/iderudit/014028ar

DOI : https://doi.org/10.7202/014028ar

Aller au sommaire du numéro

Éditeur(s)

Association Canadienne d'Ethnologie et de Folklore

ISSN

1481-5974 (imprimé)

1708-0401 (numérique)

Découvrir la revue

Citer cet article

Lukić, N. G. (2005). La représentation des immigrants dans l'espace muséal et patrimonial de Québec. Ethnologies, 27(1), 223-243.

https://doi.org/10.7202/014028ar
Résumé de l'article

Jusqu'à quel point et de quelle manière les immigrants d'hier et d'aujourd'hui partagent-ils l'espace public culturel au Québec ? Les enjeux de l'exclusion/inclusion sont rythmés par l'évolution de la définition même de l'identité québécoise et du patrimoine qui la symbolise. Comment les immigrants sont-ils représentés : d'une manière individuelle ou/et collective ? Sont-ils intégrés ou séparés, passifs ou actifs, sont-ils liés plutôt à leur communauté d'origine ou à leur expérience québécoise ? Par l'analyse des expositions Mémoires et Le Temps des Québécois, portant sur le thème de l'identité, présentées au Musée de la civilisation de Québec, cet article se propose d'interroger les représentations des immigrants et les stratégies actuelles de la médiation interculturelle.
Ce document est protégé par la loi sur le droit d'auteur. L'utilisation des services d'Érudit (y compris la reproduction) est assujettie à sa politique d'utilisation que vous pouvez consulter en ligne.

https://apropos.erudit.org/fr/usagers/politique-dutilisation/ 


\title{
LA REPRÉSENTATION DES IMMIGRANTS DANS L'ESPACE MUSÉAL ET PATRIMONIAL DE QUÉBEC
}

\author{
Nada Guzin Lukić
}

Université Laval

Les immigrants font de plus en plus partie de la société québécoise et leur nombre ne cesse de croître ${ }^{1}$. Il ne s'agit pas d'un phénomène nouveau : l'immigration est au coeur de l'histoire du Québec. Mais cette histoire est-elle inscrite dans le récit patrimonial et muséal ? Jusqu'à quel point et de quelle manière les immigrants d'hier et d'aujourd'hui partagent l'espace public culturel? Quelle image des immigrants les musées présentent-ils? Ce texte s'inscrit dans l'amorce d'un projet de recherche portant sur l'intégration des immigrants dans l'espace culturel et patrimonial québécois. Cette recherche se construit autour des questions suivantes : les milieux culturels sont-ils, comme on l'entend souvent, plus ouverts à la diversité culturelle ? Cette ouverture varie-telle d'une région à l'autre? Quelle place les immigrants au Québec ont-ils dans les institutions culturelles nationales, régionales et locales? Comment les immigrants sont-ils représentés : de manière individuelle ou/et collective ? Sont-ils intégrés ou séparés ? Sont-ils liés plutôt à leur communauté d'origine ou à leur expérience québécoise? Les enjeux de l'exclusion/inclusion sont rythmés par l'évolution de la définition même de l'identité québécoise et du patrimoine qui la symbolise. Par l'analyse des expositions portant sur le thème de l'identité, essentiellement Mémoires et Le Temps des Québécois, présentées au Musée

1. Selon les données établies par la Direction de la population et de la recherche sur la citoyenneté et l'immigration, du Ministère des relations avec les citoyens et l'immigration, le Québec a admis en moyenne 35000 immigrants par an, soit 176394 immigrants, entre 1999 et 2003. Le Ministère prévoit que ce nombre passera à 44400 en 2005 et à 48000 en 2007 (Ministère des relations avec les citoyens et l'immigration, mai 2004). 
de la civilisation de Québec, nous proposons d'interroger les représentations des immigrants et les stratégies actuelles de la médiation interculturelle.

Le Québec est la seule société francophone en Amérique du Nord et la langue demeure le trait qui la distingue le plus de ses voisins. La culture québécoise repose sur des racines françaises jointes à un héritage autochtone, à une influence de ses voisins canadiens, des États-Unis, ainsi que des immigrants de diverses origines. On souligne souvent que c'est un pays d'immigration car depuis la colonisation, le territoire a été convoité par les habitants venus d'outre-mer. Le Québec constitue une société distincte par rapport à l'Amérique du Nord et par rapport à sa culture européenne d'origine. Cette société possède des traits culturels uniques comme la langue, l'art, le patrimoine, les mœurs et, comme toute autre société minoritaire aujourd'hui, elle est fragile, à la fois enrichie et menacée par les autres cultures.

Dans le domaine de la diversité culturelle, le Québec a vécu une évolution accélérée depuis la Révolution tranquille, notamment depuis l'Exposition universelle de 1967 qui a une place importante dans l'histoire interculturelle du Québec. Cette exposition «a modifié les frontières symboliques entre Nous et les Autres»(Curien 2003: 3$)^{2}$. L'impact de l'Expo 67 sur la société québécoise est multiple dans ces années où le Québec s'est ouvert à l'autre. En effet, les expositions, comme celle de 1967 qui a marqué toute une époque, mais également celles de portée moins spectaculaires présentées dans les musées nationaux, sont des terrains propices pour les recherches interculturelles. Étant donné que la définition qu'une société se fait d'elle-même se transpose dans ses institutions culturelles, le musée en tant qu'institution de conservation et de transmission du patrimoine participe à la construction de l'identité culturelle d'un lieu, d'un pays, d'une région.

En tant que lieu public, le musée est un lieu de la représentation de la société et de ses composantes; il est aussi un espace de médiation interculturelle et de contact (voir Clifford 1997 : 188-219). La notion de représentation concerne les pratiques et les formes culturelles au moyen desquelles les sociétés interprètent et décrivent le monde autour

2. L'impact de l'Expo 67 sur la société québécoise a suscité de nombreuses recherches, dont celle de Pauline Curien, entièrement consacrée à l'analyse des représentations identitaires. Selon celle-ci, « l'Expo 67 a fourni l'occasion d'une catharsis constitutive de l'identité québécoise moderne " (2003: 2). 
d'elles et se présentent à d'autres. Elle implique un ensemble de manifestations ou de productions culturelles, comme les expositions, par lesquelles des significations sont constituées et communiquées. De telles pratiques et de telles formes culturelles se produisent et diffusent des significations au sein des groupes sociaux. On peut dire que le musée et le patrimoine sont des formes de production culturelle et des espaces de communication interculturelle.

\section{Histoire de l'immigration à Québec : une réalité à découvrir et à mettre en valeur}

La ville de Québec est généralement présentée comme une ville monoculturelle, le bastion de la culture francophone en Amérique du Nord. Ce n'est pas un hasard si l'on y trouve le Musée de l'Amérique française. Malgré ce fait incontestable, l'immigration n'est pas un phénomène nouveau dans la ville de Québec. Depuis le premier établissement dans le cœur historique de la ville et malgré la forte prédominance des francophones à diverses périodes, plusieurs autres groupes et individus s'y sont installés de manière temporaire ou permanente, comme les Anglais, les Irlandais, les Chinois, etc. Par exemple, le port de Québec a été pendant une longue période, du début du XIX ${ }^{\mathrm{e}}$ siècle jusqu'à la Première Guerre mondiale, le principal port d'entrée des immigrants au Canada. Cette histoire est-elle mise en valeur? Aujourd'hui, l'unique lieu de mémoire de l'immigration mis en valeur au Québec est celui de Grosse-Île où se trouve le Mémorial des Irlandais. Ce lieu historique national du Canada valorise trois phénomènes distincts qui sont

tout d'abord, l'importance de l'immigration au Canada, plus particulièrement via la porte d'entrée de Québec, du début du XIX siècle jusqu'à la Première Guerre mondiale. Ensuite, les événements tragiques vécus par les immigrants irlandais, spécialement lors de l'épidémie de typhus de 1847 . Finalement, la commémoration du lieu repose sur le rôle joué par l'île, de 1832 à 1937, à titre de station de quarantaine du port de Québec, longtemps le principal point d'arrivée des immigrants au Canada (Lieu historique national du Canada de la Grosse-Île-et-le-Mémorial-des-Irlandais, $1^{\mathrm{er}}$ mars 2005).

La décision de mettre l'accent, dans la mise en valeur de GrosseÎle, sur la station de quarantaine du port de Québec (entre 1832 et 1937) souligne l'aspect tragique de l'immigration irlandaise. Cette commémoration n'est cependant pas accompagnée de la mise en valeur 
d'autres traces de l'immigration, comme l'installation et les vestiges de l'accueil des immigrants à Québec. Selon l'historien Alain Roy, l'histoire de l'immigration est occultée dans la ville de Québec. Le fait de mettre en valeur des vestiges uniquement à Grosse-Île peut contribuer à une image d'un Québec replié sur lui-même et hostile à l'immigration. Enfin, il ne s'agit pas de ce lieu de mémoire proprement dit, mais du fait qu'il s'agisse de l'unique lieu national de commémoration de l'immigration au Québec et que celui-ci ne montre qu'une facette du phénomène de l'immigration: maladie, souffrance, quarantaine et danger que représentaient les immigrants pour la population locale.

La problématique de l'immigration au Québec est complexe encore actuellement, tant sur le plan patrimonial que politique, car l'immigration relève du gouvernement fédéral, mais en même temps, Québec est depuis $1978^{3}$ l'unique province au Canada à avoir le droit de choisir les immigrants qui vont s'y établir ${ }^{4}$. Au Québec, l'éducation et la culture demeurent du ressort de la province. Cela dit, en matière d'immigration, particulièrement dans le domaine culturel, Québec cherche à traiter ces questions de la façon qui correspond le mieux à la réalité québécoise en tant que société distincte (Taylor 1992)5. La mise en valeur de l'histoire et du patrimoine liés à l'immigration suit un peu la même logique (voir Pâquet 1997, 2005).

Un autre fait, qui n'est pas particulier au Québec mais qui façonne l'aspect culturel de l'immigration, est que la majorité des immigrants s'établissent dans les grands centres urbains. Les villes des régions ont de la difficulté à retenir les immigrants, notamment la ville de Québec. Les raisons en sont-elles seulement économiques (le manque de travail)

3. Depuis 1978, grâce à l'entente Cullen-Couture, le Québec peut faire sa propre sélection d'immigrants, même si leur admission doit être approuvée par Ottawa.

4. Le Ministère des relations avec les citoyens et de l'immigration a changé les critères de sélection des immigrants en introduisant la langue française comme critère important, ce qui a changé considérablement la donne culturelle : pour l'année 2003-2004, la majorité des immigrants proviennent de France. Voir le tableau «Immigration au Québec selon les principaux pays de dernière résidence, $3^{\mathrm{e}}$ trimestre et 9 premiers mois 2004 » (Ministère de la Culture et des Communications $2005: 5$ ).

5. Charles Taylor propose une critique du multiculturalisme à travers la réflexion sur l'enjeu de la demande de reconnaissance par tous les groupes minoritaires au sein des sociétés démocratiques. Le livre se base sur les relations QuébecCanada ainsi que sur les relations entre le Québec et les minorités, à la lumière de la reconnaissance du Québec comme société distincte. 
ou bien également culturelles ? En effet, les centres urbains et cosmopolites comme Montréal, Toronto ou Vancouver offrent une concentration plus grande d'immigrants. L'intégration à un environnement cosmopolite et globalisé a une dynamique différente de celle qui s'effectue dans des milieux plus homogènes. Un autre aspect à retenir concerne la diversité des cultures au Québec et leur relation. Quand on évoque l'immigration (tant au niveau provincial que fédéral), la relation étudiée est généralement celle qui se produit avec l'un des groupes majoritaires, francophone ou anglophone; la relation avec les populations autochtones est rarement interrogée. Le statut particulier des Autochtones, résultant des lois sur les réserves, exclut toute immigration sur leur territoire. Par contre, sur un plan culturel, les contacts ont toujours existé.

En effet, à la base de toute étude sur les relations entre les immigrants et l'immigration réside la définition de l'immigrant que propose une société. Qui est l'immigrant? Au niveau de la loi canadienne et québécoise, l'immigrant est un résident permanent. Sur le plan juridique, avec l'obtention de la citoyenneté canadienne, il perd son statut d'immigrant. Au niveau socioculturel cependant, l'identité d'immigrant peut se perpétuer pendant plusieurs générations et elle est définie généralement par la filiation ethnique ou nationale d'origine.

Nous interrogeons l'identité attribuée à l'immigrant et l'intégration de son parcours dans le discours historique et identitaire du Québec, celui qui est énoncé dans les expositions ou les écrits qui ont servi à sa conception et à sa diffusion (dépliants, catalogues, sites Internet des musées, etc.). Quelle définition de l'immigrant les institutions culturelles proposent-elles, notamment le musée ? Comment les relations avec l'immigrant se construisent-elles et sont-elles représentées dans le récit muséal ? La définition contemporaine du "Québécois » est inclusive : nous verrons comment les institutions véhiculent et représentent ce discours.

Le choix du Musée de la civilisation comme la première institution à aborder se justifie par plusieurs raisons. D'abord ce musée d'État ${ }^{6}$, en tant que musée national, a une mission claire, qui est de

6. Au Québec, il y a trois musées nationaux : le Musée d'art contemporain de Montréal, le Musée national des beaux-arts du Québec et le Musée de la Civilisation, ces deux derniers se trouvant à Québec. 
faire connaître l'histoire et les diverses composantes de notre civilisation, notamment les cultures matérielle et sociale des occupants du territoire québécois [...], assurer la conservation et la mise en valeur de la collection ethnographique et des autres collections représentatives de notre civilisation (Lois et règlements du Québec, $1^{\text {er }}$ décembre 2004).

En effet, le Musée de la civilisation a pour mandat de conserver et de mettre en valeur la culture des Québécois de toutes origines et, en tant que musée d'État, il a une responsabilité sociale. Sa fondation en 1988, sous le nom de Musée de la civilisation ${ }^{7}$, énonce déjà son orientation. Il se veut plus ouvert et consacré à l'ensemble des phénomènes sociaux. Le fondateur et premier directeur du Musée explique ce choix par la diversification de la société québécoise dans les années 1970-1980.

Traditionnellement de provenance européenne, la masse migratoire se modifie. La blanche et très chrétienne Europe n'offre plus qu'une maigre part des nouveaux arrivés. [...] La polarité anglais/français, protestant/catholique qui définissait les paradigmes du nationalisme ethnique canadien-français glisse irrévocablement vers une nouvelle conception de la nation, plus ouverte à d'autres aspects culturels (Arpin 1998: 12).

Cette évolution provoque une crise d'identité et des changements dans la définition de la nation.

Ces changements influencent les choix culturels, y compris celui de créer un Musée de la civilisation ancré sur le territoire québécois et ouvert à tous les citoyens de quelque origine qu'ils soient (Arpin 1998: 13). La société québécoise a changé davantage ces dix dernières années ; les négociations politiques, identitaires et culturelles avec les Autochtones et avec les communautés culturelles sont nombreuses. Le projet social et culturel québécois se veut de plus en plus ouvert; le concept d'une nation civique émerge dans le discours des historiens et des hommes politiques. Enfin, en soi, la fondation du Musée en tant que projet de civilisation participe de l'émergence d'un nouveau type de musée, le musée de société, qui inclut la philosophie postmoderne d'un environnement plus éclaté.

7. Au début du projet, le nom du Musée était le «Musée de l'Homme d'ici ». 
Au sein du complexe du Musée de la civilisation ${ }^{8}$, il y a plusieurs expositions identitaires: Mémoires (1988), Amérique française et Nous, les premières nations (1998), Le Temps des Québécois (2004) et l'exposition virtuelle Identités américaines ${ }^{9}$ (2000). Chacune à sa manière expose une facette de l'identité québécoise. Ainsi, par exemple, l'exposition Amérique française nous invite à découvrir comment la culture française s'est étendue sur le territoire nord-américain et comment elle s'y est enracinée (Musée de l'Amérique française, 3 mars 2005). Cette exposition est consacrée à une identité culturelle dont le trait d'identification est la langue. Dans la perspective de l'immigration récente des immigrants francophones, plus nombreux depuis le changement des critères de sélection de l'immigration, quand la langue devient le critère important, on peut se demander où s'arrête "l'histoire consacrée à la commémoration de la culture française en Amérique » (Bergeron 1996: 100) ? Certes, cette culture française au Musée de l'Amérique française est basée sur la collection des prêtres du Séminaire de Québec, mais elle s'est enrichie aussi des acquisitions contemporaines. Il serait significatif d'étudier les choix de l'institution concernant ces acquisitions. Quels objets sont ajoutés à cette collection afin de continuer à répondre au mandat et à la mission de l'institution sur les francophones en Amérique du nord ? Les collections se sont-elles arrêtés à une période donnée (dans ce cas, elles complèteraient la collection existante), ou prétendent-elles s'aventurer jusqu'aux périodes récentes? En effet, la position du Musée est ouverte dans ce sens.

Le concept du Musée de l'Amérique française s'ouvre largement à l'apport des groupes culturels qui marquent l'Amérique d'une saveur française. S'il limite son territoire à l'Amérique du Nord, il n'en referme pas les portes. Dans son ouverture, il porte, par contre, une attention particulière à l'immigration francophone récente au Québec (Arpin 1998 : 101).

La problématique du collectionnement des traces de l'immigration se pose de plus en plus et risque de s'accentuer dans les institutions muséales. Relevant plus des mémoires de pratiques et des savoir-faire

8. Le Musée de l'Amérique française et le Centre d'interprétation de la PlaceRoyale font partie du complexe du Musée de la civilisation.

9. L'exposition Identités américaines propose la question «Que signifie être américain ? » à huit musées « des quatre coins des Amériques » (Musées et millénaire, 3 mars 2005). 
que de la culture matérielle, les traces liées à l'expérience de l'immigration posent un défi aux institutions. La récente politique des collections fait état de cette situation.

L'épopée de ces Québécois d'origines diverses mérite d'être documentée, conservée, racontée. [...] S'il est plus évident de conserver les objets des premières vagues d'immigrants, tout simplement parce qu'ils sont devenus nôtres, la conservation du souvenir des nouveaux arrivés est moins facile. Elle exige une démarche proactive de recherche et de mise en place de collaboration. Elle demande également de faire usage de nouvelles techniques de documentation (documents visuels, audio, etc.) (Gendreau 2003 : 51).

On se rend compte que le discours sur l'intégration reste lettre morte s'il ne s'accompagne pas des mesures pour le mettre en oeuvre. Dans le cas de la pratique muséale, l'immigration commence à être considérée d'une manière plus organisée comme thème, comme champ de recherche et de collectionnement.

\section{Les Mémoires des Québécois}

L'exposition qui a particulièrement attiré notre attention est Mémoires, car elle permet « de découvrir des souvenirs collectifs autour desquels s'est forgée l'identité culturelle des Québécois » (Musée de la civilisation 2005a). Elle aborde les trois thèmes suivants : mémoire collective, identité et histoire. Quelle place donne-t-elle et quelle identité attribue-t-elle aux immigrants? Les sources primaires utilisées pour analyser cette exposition sont les éléments offerts aux visiteurs : les écrits dans l'exposition et le matériel qui l'accompagne, comme les dépliants ou le site Internet du Musée.

Ces Mémoires retracent les souvenirs marquants de l'histoire et de la culture du peuple québécois. Il s'agit de " la première grande synthèse de l'histoire du Québec traitée par le biais des émotions » (Mémoires, 2005). Le panneau d'introduction annonce une exposition sur l'identité culturelle, soulignant que chacun participe différemment au façonnement d'une identité plurielle. L'exposition couvre plus de quatre siècles, «depuis l'arrivée des Français jusqu'aux enjeux de l'heure ». Elle offre «la possibilité d'élaborer différentes manières de se souvenir, en faisant écho indirectement à la devise nationale, Je me souviens, qui devenait tout-à-coup, vue sous cet angle, multiple»(Dubé 1991 : 32). 
L'identité québécoise est présentée à travers différents types de mémoires : mémoire nostalgique, mémoire des adaptations, mémoire refoulée, mémoire obligée, mémoire libre et mémoire bilan. Ainsi, par exemple, la zone de mémoire d'adaptation est divisée en trois parties : vivre de la nature, les Autochtones et les arrivants, leur relation caractérisée par les emprunts. Les Québécois, pour survivre, ont emprunté les moyens et les techniques des Amérindiens. À la fin du XVIII ${ }^{\mathrm{e}}$ siècle, on trouve un autre groupe, les Loyalistes, qui reçoivent des terres dans les Cantons de l'Est. Les immigrants sont cependant concentrés à Montréal. Avec l'urbanisation, "Montréal au XX ${ }^{\mathrm{e}}$ siècle devient cosmopolite » et le texte nous explique la composition de la ville.

Les communautés française, anglaise et irlandaise s'y côtoyaient déjà et la ville accueille, durant les trois premières décennies, plus de 120000 immigrants européens, d'origine juive surtout. L'apport des autres groupes ethniques est plus récent. Aujourd'hui, on compte environ 100 groupes d'origines ethniques différentes au Québec (Musée de la civilisation 2005).

La dernière zone de l'exposition est la plus significative au terme de notre investigation. Intitulée «Et maintenant?», elle propose une mémoire bilan. En effet, malgré l'approche par mémoires, l'exposition suit une trame chronologique en se terminant avec le Québec contemporain et annonce une culture riche et éclatée des Québécois (cirque, théâtre, littérature). «Notre culture » est représentée par des témoignages de succès de Québécois : ingénieurs, artistes, entrepreneurs comme Bombardier, etc. Selon les témoignages, la culture québécoise est menacée plutôt de l'extérieur, par la mondialisation et l'uniformisation de la culture. Les « success stories » mises de l'avant mettent en lumière les Québécois pure laine francophones. Plusieurs critiques, comme on le verra plus tard, considèrent cette exposition comme nationaliste, car les mémoires sont exclusivement francophones, avec peu de présence des anglophones et des Autochtones.

L'identité québécoise est interrogée dans la dernière zone, à travers des entrevues et des témoignages présentés en vidéo. «Nos gens » sont identifiés selon diverses catégories : les Québécois de vieille souche, les nouveaux arrivants, les «pure laine » et les néo-Québécois. La sélection des personnes est faite de façon à briser les stéréotypes sur les identités immuables. Ainsi, à la question «Suis-je pure laine? ?, les réponses ne proviennent pas seulement de Québécois francophones catholiques et 
blancs, mais aussi de gens d'origine mixte qui se sentent «pure laine »: Henriette, d'origine mixte francophone et amérindienne, qui se sent amérindienne "pure laine »; ou Steven, d'origine anglophone qui se sent Québécois «pure laine » mais pas séparatiste ; Jean, qui considère le Québec comme «ma patrie et le plus beau pays »; ou Allan, qui a un nom anglophone mais qui est "pure laine » québécois francophone. Les néo-Québécois sont représentés par les réfugiés politiques du Rwanda ou du Cambodge, Ingrid d'Haïti, le professeur d'anglais Mel, ou Gina, qui est née au Québec mais de parents d'origine italienne. La zone "Intolérants/tolérants » sonde ces deux types de relations. Les témoignages sont symptomatiques de la situation, tant à la fin des années 1980 qu'aujourd'hui : le racisme, notamment envers la minorité visible «qui ne veut pas s'adapter», les témoignages de l'ambivalence, ou de l'enthousiasme envers les immigrants décrits comme «du beau monde » et la richesse des contacts interculturels. Cette zone montre une ouverture vers les identités et les mémoires multiples et une culture de convergence (sur ce concept de convergence, voir Mathieu et Lacoursière 1991 : 114-131).

Malgré le succès indéniable de cette exposition, analysée entre autres lors du colloque qui lui a été consacré en 2004, la difficulté de la remplacer démontre toute la complexité d'une présentation de l'histoire nationale dans une société en mutation et de plus en plus multiculturelle. Il s'agit d'une exposition-référence de la muséologie québécoise ; son apport est considéré comme indéniable, tant par les professionnels que pour le public, bien qu'elle ait été créée en 1988 et qu'elle n'ait été que légèrement modifiée depuis.

Cependant, elle est critiquée, surtout pour « la faible présence des “autres” que les Québécois» (Sauvage 2004: 42)10, ainsi que pour le fait qu'elle ignore les apports de l'immigration (76). En 1998, le Musée tente de corriger cette lacune par l'exposition Nous, les premières nations, qui se veut le contrepoids de l'exposition Mémoires, avec le même nombre d'objets (500), avec la consultation des Autochtones, etc. Ces critiques montrent que les attentes sont grandes envers un tel musée ayant pour mandat de couvrir toute une société dans son passé, son présent et son avenir. Chaque groupe vise la reconnaissance et sa part sur la place publique. L'exposition Mémoires a été à l'avant-garde à la fin des années 1980 en termes muséologiques certes, mais aussi par sa

10. Les Québécois désignent ici ceux de souche francophone. 
proposition d'une culture québécoise de convergence qui se nourrit des contacts et des interactions. Très riche, elle offre plusieurs niveaux de lecture. Enfin, les expositions permanentes sont le fruit d'un long processus, mais c'est seulement une petite partie de la recherche qui est transposée dans l'espace. L'exposition Mémoires est exemplaire en ce sens. L'ouvrage déjà mentionné de Mathieu et Lacoursière, Les mémoires québécoises (1991), issu de la préparation de l'exposition, offre une analyse approfondie de la population québécoise, de sa composition et de ses relations internes, proposant le concept de culture de convergence par l'apport des divers groupes culturels à la société québécoise ${ }^{11}$. Toutefois, l'exposition reflète le groupe culturel majoritaire, en mettant en avant davantage les mémoires des régions, monoculturelles, que le cosmopolitisme et le multiculturalisme de Montréal, par exemple. Mais elle a suscité les émotions des visiteurs, et c'est certainement en cela qu'elle a le mieux réussi. On peut dire que la médiation interculturelle est basée ici sur l'émotion. Pour les Québécois, l'émotion offre la confirmation de leur identité culturelle et, pour les nouveaux arrivants, elle fait germer la création du lien avec le nouveau pays.

L'approche proposée peut être qualifiée d'inclusive. Par contre, la mise en espace reflète cette approche d'une manière inégale, car le thème de l'immigration est intégré au récit historique et mémoriel national, essentiellement toutefois dans la dernière zone des Mémoires. La définition du «Québécois » dans le discours des deux expositions analysées inclut les Québécois de toutes origines. Une telle définition n'est cependant pas facile à réaliser dans la mise en espace de l'exposition, vu qu'il s'agit aussi d'une négociation de l'espace physique et du nombre d'objets associés à chaque composante identitaire du récit. Nous examinerons cette inscription physique des immigrants dans une autre exposition permanente du Musée de la civilisation, inaugurée en juin 2004 : Le Temps des Québécois.

\section{Le Temps des Québécois}

Le Temps des Québécois raconte une histoire «qui appartient à tous ceux qui ont habité et construit le Québec » (Musée de la civilisation 2005b). Les Québécois sont plutôt identifiés par leur contribution à la

11. Le troisième chapitre, "La population québécoise : composition et interrelation ", développe plus précisément ce concept de la culture de convergence (Mathieu et Lacoursière 1991 : 97-132). 
construction du pays. La diversité culturelle est énoncée ainsi : « hommes et femmes, célèbres ou anonymes, autochtones, francophones, anglophones ou allophones, catholiques, protestants ou juifs, qui ont relevé la tête, retroussé leurs manches, lutté, créé, entrepris, bâti, fondé, travaillé, récolté pour façonner le Québec d'aujourd'hui » (Musée de la civilisation 2005b). Dans le catalogue de l'exposition, on trouve la confirmation de cette interprétation de l'identité québécoise par un investissement participatif : "lorsque nous employons le terme "Québécois", nous [ne faisons donc pas référence] aux seuls francophones, mais à tous ceux qui ont investi, dans l'édification historique de cette société, leurs labeurs et leurs aspirations» (Létourneau 2004: 6). Ce discours est-il transposé dans l'espace de l'exposition? Comment se traduit-il ?

L'exposition est annoncée ainsi.

De la Nouvelle-France à aujourd'hui, parcourez la grande et la petite histoire du Québec. Venez vivre les élans et les replis, les réussites et les difficultés, la ferveur et la froidure de cette histoire qui appartient à tous ceux qui ont habité et construit le Québec (Musée de la civilisation 2005b).

Le panneau d'introduction nous invite à ouvrir un vieux livre. L'exposition a une facture traditionnelle dans son approche et dans sa muséographie. On ne procède plus par mémoires mais par faits historiques; on ne vise plus à partager des émotions mais des connaissances. Le visiteur entre dans un cours d'histoire. La chronologie plus que l'approche thématique, la culture matérielle, les documents et les objets ${ }^{12}$ illustrent les périodes et les événements sélectionnés et expliqués par les textes, les films de reconstitution historique et les récits des historiens. La ligne du temps rythme l'axe central. La trame chronologique est divisée en quatre périodes : de 1608 à 1760, de 1760 à 1840, de 1840 à 1960 et de 1960 à aujourd'hui. Ce «Fil de l'histoire politique », de la Nouvelle-France à aujourd'hui, est la trame de l'exposition. Pour faire ce tour historique du Québec, «les visiteurs sont invités à revivre des événements, à rencontrer des personnages d'autrefois, à admirer des objets de collection et à prendre contact avec un passé révolu mais fondateur» (Musée de la civilisation 2005b). Selon la directrice du Musée, l'exposition montre aussi « le visage

12. Comme pour l'exposition Nous, les premières nations, plus de 500 objets servent de support à ce récit. 
pluriculturel de la société québécoise » (Musée de la civilisation 2005b). Enfin, l'exposition présente, tout au long du parcours, les témoignages sonores d'une cinquantaine d'hommes et de femmes qui, avec leurs rêves et leurs aspirations, ont vécu les défis et les événements de leur époque. On trouve aussi, parmi «les voix surgies du passé », celles des immigrants : la famille O'Brien pour les Irlandais et la famille de Nicola Manzoni pour les Italiens. Leur point commun est qu'ils ont « fui la misère » pour s'installer au Québec. La sélection est représentative en termes identitaires et inclut quelques personnages autres que des catholiques francophones : il y a Kondiaronk, chef amérindien signataire de la Grande Paix, Ezechiel Hart, commerçant juif de Trois-Rivières, etc.

Le film documentaire intitulé Le Québec en mouvement? de Benoît Pilon, présenté à la fin de l'exposition, propose une réflexion sur certains problèmes ou questionnements de la société québécoise actuelle. Les thèmes de l'immigration, du vieillissement de la population, de la santé et de la mondialisation sont développés à travers les témoignages recueillis dans la rue, à Montréal et à Québec, et les réflexions d'un historien, d'un sociologue, d'un écrivain immigrant, entre autres. Ainsi, on s'interroge sur l'identité ${ }^{13}$ et la relation avec les immigrants de plus en plus présents dans la société québécoise. Toutefois, les «minorités visibles » ont été filmées essentiellement dans les rues de Montréal. On évoque le contrat moral avec les nouveaux arrivants et les valeurs québécoises non négociables : la langue française et les lois. La différence entre l'immigration au Québec et ailleurs au Canada est présentée à travers le témoignage d'un immigrant qui nous dit qu'ailleurs au Canada, on est obligé de garder sa culture d'origine et qu'au Québec, on parle plutôt d'intégration dans l'optique d'une nation civique et inclusive. Malgré le discours qui se veut inclusif tout au long de l'exposition et le film qui fait un tour des éléments principaux et des opinions divergentes sur la question, une vitrine à la fin de l'exposition, «Les multiples visages », témoigne d'une autre réalité de l'immigration dans le Québec actuel : celle de la marginalité de la question et des stéréotypes existants. Ce qui nous amène à la question de la véritable place des immigrants dans l'espace culturel québécois. Déjà la place physique de cette vitrine, située après la fin de l'exposition, dans le corridor peu éclairé qui mène à la sortie, interpelle et provoque un véritable malaise auprès de certains

13. Lors du tournage de ce film la question suivante a été posée aux gens de la rue : «Que signifie pour vous être Québécois aujourd'hui ?» 
visiteurs, notamment des immigrants. Il est évident que l'exposition aurait pu se terminer où elle se termine vraiment: avec l'Expo 1967, les Jeux Olympiques de Montréal en 1976 et la vitrine « Je vous entends chanter », consacrée à la chanson québécoise. Elle contient une dizaine d'objets présentant les immigrants du Québec moderne. Quels objets ? Un bonnet du Portugal portant les couleurs du drapeau du Portugal (1988), une darbouka d'Afrique du Nord, un tambour typique des cultures arabes, une babouche de femme d'Afrique du Nord (XXe siècle), une mallette «cot-cot» du Sénégal, fabriquée vers 1975 avec des matériaux de récupération (boîtes de conserves) et présentée comme un exemple de la créativité de "l'économie informelle africaine ", un collier de Tunisie, une cithare à seize cordes de Saigon (1970) avec le texte suivant: "Après la chute de Saigon, les Vietnamiens ont fui le régime communiste. Plusieurs se sont installés au Québec. L'un d'eux apporta cette cithare. Malgré la douleur de l'exil, sa musique lui donna foi et courage ». Tout est là : l'image de l'immigrant joyeux malgré son malheureux destin, la vaisselle et le menu du premier restaurant chinois Sam Wong ouvert à Sainte-Foy à Québec en 1963, une lampe d'Haïti, etc. Le texte explicatif de la vitrine «Les multiples visages » nous dit que les immigrants proviennent surtout des pays du Sud « où sévissent les crises économiques et politiques ", et que le Québec exerce sa compétence en matière d'immigration et redouble d'efforts pour accueillir et intégrer les immigrants. "Le visage du Québec rajeunit et se nuance ». Les objets exposés et le texte qui les accompagne font partie de l'iconographie de l'immigration et de la diversité culturelle habituelle. Mais le Musée ne peut-il pas aller plus loin ?

Le discours officiel véhiculé par les historiens, les sociologues, les écrits sur l'immigration, notamment dans le film qui se termine avec le slogan : "être Québécois aujourd'hui ça signifie peut-être seulement qu'on accepte de dire : "je suis ici chez-moi ${ }^{14}$ !" », se veut inclusif. Cette fin invitante termine l'exposition sur l'histoire du Québec et montre une ouverture suggérant un lien d'identification et un ancrage ici (le territoire québécois). Ce film pose aussi des questions importantes sur la complexité des négociations identitaires et culturelles du Québec d'aujourd'hui. Comment expliquer le décalage entre cette réflexion nuancée et la sélection simpliste des objets réduite aux stéréotypes : restaurant ethnique, musique, artisanat reconnaissable calqué sur le souvenir de voyage dans les pays exotiques (collier de Tunisie), etc. ?

14. Benoît Pilon, scénariste et réalisateur du film «Le Québec en mouvement». 
La lecture de cette vitrine nous propose la perception suivante des immigrants au Québec: des gens malheureux et pauvres qui jouent d'instruments ethniques pour soulager leur peine et leur chagrin provoqué par l'exil et dont l'apport majeur à la société québécoise réside dans les restaurants ethniques. Il reste toutefois qu'un regroupement d'objets permet des lectures multiples, qui ne dépendent pas seulement du concepteur mais aussi des perceptions du visiteur. Certes, il faut une certaine sensibilité à la question de l'immigration pour en faire une telle lecture. Malgré cette mise en garde et compte tenu de la subjectivité du visiteur, il reste qu'on observe, sinon un double discours, du moins un manque de profondeur de la réflexion sur l'immigration dans cette exposition qui, tout au long, dans les autres propos, a su montrer profondeur et nuance. Plutôt que de présenter les objets stéréotypés liés essentiellement au pays d'origine, étant donné que le propos de l'exposition est l'histoire du Québec, ou encore d'évoquer la mémoire nostalgique des immigrants, il aurait été plus pertinent de présenter leur contribution ou leur lien avec le Québec. Le problème de ces représentations est qu'on ne voit pas ce lien, à l'exception de leur simple et passive présence ici. On aurait plutôt pu montrer ce que ces immigrants peuvent nous apporter, leur contribution à l'histoire québécoise, car c'est de cela qu'il s'agit dans cette exposition et non pas de la représentation du folklore des habitants de diverses origines du Québec.

Comment expliquer ce double discours de l'exposition? Plusieurs hypothèses peuvent être formulées. On pourrait en conclure que le film représente l'immigration montréalaise, une réalité plus présente et prise en considération, tandis que la vitrine «Les multiples visages du Québec » représente l'immigration dans la ville de Québec où, en fait, il y a peu d'immigrants et où ce phénomène est marginalisé. L'hypothèse qui répond le plus à la réalité est que cette représentation reflète le traitement de cette question au Québec. On y trouve un discours savant et officiel de l'inclusion, tant identitaire que culturelle et, en parallèle, la situation sur le terrain, notamment à Québec. On peut la décrire comme la marginalisation de cette question dans les institutions culturelles, qui donnent peu d'espace à la médiation interculturelle et où l'on trouve encore des stéréotypes, compte tenu de la perception de l'immigrant, de ses caractéristiques et de sa relation avec la société d'accueil. L'immigrant est identifié essentiellement par sa filiation ethnique ou son pays d'origine ; sa vie québécoise est généralement 
inexistante. Présenter l'histoire québécoise de l'immigration permettra à l'immigrant de première, voire de deuxième ou troisième génération de s'identifier avec la société d'accueil et aux membres de la société d'accueil, de mieux percevoir l'expérience de l'immigration. La mémoire de l'adaptation est au moins aussi importante que la mémoire nostalgique du pays d'origine. Présenter l'immigration au musée propose un défi de taille qui dépend plus qu'on ne le pense de la définition de l'identité qu'une société propose d'elle-même. Le Québec se veut aujourd'hui un pays d'accueil ouvert qui tient cependant à préserver les caractéristiques qui font sa particularité culturelle dont les éléments de base sont: la langue, la démocratie et le respect de l'autre. Il n'est pas difficile d'adhérer à une telle proposition sociale. Toutefois, comment surmonter le décalage entre le discours et sa représentation muséale et patrimoniale?

La politique de l'interculturalité soutenue sur la scène politique, médiatique et culturelle québécoise depuis les années 1990 laisse nécessairement des traces dans les pratiques et les manifestations culturelles. Le Musée de la civilisation a le mandat d'élaborer ces questions au moyen des activités et des expositions, mais encore de manière ponctuelle et sporadique. Ainsi, par exemple, il servait de vitrine médiatique lors de l'accueil des réfugiés kosovars en 1999; il a lancé la semaine de l'histoire des Noirs en 2004, etc. Quelques expositions temporaires qui interrogent la diversité et le métissage, même si ce n'est pas de manière directe, peuvent s'inscrire aussi dans la problématique de l'immigration. Mais l'unique exposition entièrement consacrée à ce thème fut Les immigrants racontent, présentée du 15 novembre 1996 au 19 octobre 1997. Cette exposition était basée sur les histoires de vie des immigrants vivant au Québec.

Les évolutions ne sont pas linéaires; les identités se composent et se recomposent et leur traitement change continuellement. Enfin, la dernière zone de l'exposition Mémoires ouverte en 1988 pose la question de manière plus poussée en sortant des stéréotypes folkloriques présentant les diverses capsules avec les témoignages des Québécois de diverses origines et leur regard sur l'identité québécoise. À la fin de Mémoires, la question de la diversité culturelle, de l'immigration et des constructions identitaires est abordée de manière enthousiaste, montrant une ouverture sur l'avenir incertain mais riche de possibilités dans un décor de modernité (les écrans et les moniteurs blancs énonçant une société de communication). En effet, à la fin des années 1980, l'utopie 
du progrès était encore au rendez-vous. Mais ces temps sont révolus et l'exposition proposée en 2004, dans un nouveau millénaire désillusionné, est plus sérieuse, se réfugiant derrière la scientificité du propos et la neutralité de l'institution muséale. Plusieurs mythes ${ }^{15}$ occultent la représentation des immigrants : leur inscription dans la notion d'origine initiale; le fait que la société d'accueil tolère l'immigré seulement s'il reste dans son statut d'immigré et le fait que l'immigration soit identifiée à un problème social.

On peut penser toutefois que les actions du Musée seront de plus en plus articulées en matière d'immigration, compte tenu de la situation de plus en plus multiculturelle du Québec et des tendances actuelles concernant l'intégration des immigrants dans l'offre muséale internationale, notamment par la création des musées de l'immigration. Le Musée de la civilisation montre une volonté, selon la communication de la représentante du Musée au colloque sur les musées de l'immigration.

L'immigration représente pour nous un défi majeur. [...] La pluralité culturelle est en effet un enjeux déterminant pour le Québec, car nous devons réussir l'intégration des immigrants au difficile contexte linguistique et culturel nord-américain... [Le Musée de la Civilisation] peut d'une part favoriser l'accueil et l'ouverture des citoyens à tous ceux et celles qui ont récemment choisi de devenir Québécois et, d'autre part, contribuer au développement d'un sentiment d'appartenance des immigrants envers leur société d'accueil [...] de participer à la construction d'une identité collective que nous inscrivons à notre programmation des activités qui traitent de l'immigration déjà faite, et même davantage, l'immigration qui se fait. Si l'un est tourné vers le passé et fait l'appel à la mémoire et à l'histoire, l'autre est en cours de réalisation et s'élabore dans le croisement des regards (Gendreau 2004 : 36).

Enfin, le Musée de la civilisation vise de plus en plus à relever le défi de la présentation des immigrants et de leurs histoires. Toutefois, on peut se demander si l'approche identitaire est l'unique façon de présenter la diversité culturelle d'un pays, d'une ville ou d'une région. Faut-t-il toujours isoler les identités culturelles et politiques ? L'approche identitaire n'est-elle pas en même temps réductrice d'une réalité plus complexe des identités? Une approche plurielle qui tiendrait compte

15. Nous empruntons en partie cette idée à Baccouche Naser (communication prononcée lors du premier séminaire d'Observatoire pancanadien de l'immigration en novembre 2004 à l'Université de Sherbrooke). 
des interactions et influences réciproques ne serait-elle pas même plus pertinente? La muséologie et la muséographie actuelles tendent à la création de musées d'immigration ; ce sont en fait des institutions ayant pour thème une identité sociale et culturelle, notamment dans les pays d'immigration : Ellis Island Immigration Museum à New York (1999), Immigration Museum à Melbourne (1998), mais aussi dans les Étatsnations comme la France, avec son projet actuel du Musée de l'immigration à Paris.

Les dynamiques identitaires sont analysées par rapport au centre et à la périphérie ; l'ouverture et la fermeture, l'inclusion et l'exclusion sont ainsi la manière la plus utilisée pour décrire ces situations. Outre cette analyse basée sur la polarisation et la dichotomie, les approches des médiations interculturelles offrent des possibilités plus nuancées, plus fluides et proposent des possibilités et des pratiques inattendues des relations qui se manifestent dans le champ culturel. Nous avons limité l'analyse aux expositions historiques et identitaires afin d'interroger la présence et l'intégration de l'histoire de l'immigration dans ce discours. Le développement des recherches et des expositions sur l'histoire de l'immigration, peu exploitée dans les musées actuellement, permettrait une meilleure compréhension de ces dynamiques, un réexamen et une relativisation des relations entre centre et périphérie. L'importance du Québec comme port d'entrée de l'immigration au XVII siècle, par exemple, est un fait peu connu en dehors des cercles d'historiens, puisqu'il n'est pas mis en valeur comme les autres lieux de mémoires de la Ville de Québec. La mise en valeur des traces liées à l'immigration donnera une autre facette à cette ville et à la perception de l'immigration présentée le plus souvent comme un phénomène récent. L'intégration des immigrants dans un espace culturel homogène en dehors des centres cosmopolites et globalisés est le défi de toute politique de protection de la diversité culturelle : protéger et mettre en valeur les diverses traces ou manifestations culturelles sans qu'elles représentent une menace mutuelle. La question de la négociation et encore davantage des médiations interculturelles se trouve ainsi au centre tant des théories que des pratiques de telles réalisations.

La reconnaissance de l'histoire de l'immigration sur la place publique permettra de mieux connaître la complexité de la société québécoise contemporaine, ses aspirations, ses conflits et ses évolutions. Elle contribuera à donner du sens à ces phénomènes au sein du Québec et 
du Canada. Étudier et mettre en valeur l'immigration dans une perspective historique, notamment en dehors de Montréal, offrira d'autres perspectives que celle de l'option cosmopolite qui correspond à une ville d'environ trois millions d'habitants. Le Musée de la civilisation se prête plus que tout autre musée du Québec à approfondir et à expérimenter le fait de l'immigration, comme il l'a par ailleurs déjà fait à quelques reprises par les expositions temporaires. Il reste cependant que ce volet, malgré sa présence dans la philosophie et le mandat du musée, n'est pas encore suffisamment exploité. Le temps est peut-être venu qu'après Mémoires, qu'après Nous, les premières nations et Le Temps des Québécois, nous ayons Nous, les immigrants québécois.

Quoiqu'il en soit, le manque d'approfondissement de la problématique de l'immigration, notamment l'immigration récente, dans ces présentations muséales par identités est évident. Il nous semble important d'étudier les diverses perceptions et rapports au patrimoine du pays d'accueil, puisque le processus d'adaptation sociale dans un nouveau pays est indissociable de l'adaptation culturelle. Ce processus d'adaptation culturelle nécessite une médiation, une interprétation adaptée et accessible du patrimoine culturel du pays d'accueil. Certes, la médiation qui vise l'appropriation de la culture dans son sens le plus large facilitera l'adaptation sociale et culturelle. Le musée en tant qu'institution de transmission du patrimoine participe à la construction de l'identité culturelle d'un lieu, d'un pays, d'une région. L'interprétation, la présentation et la transmission de l'histoire et du patrimoine se réactualisent sans cesse. Le musée en tant qu'espace public de l'art et du patrimoine est le lieu où la médiation culturelle et interculturelle se réalise. Certes, chaque ensemble culturel doit se différencier des autres, mais une ouverture entre groupes ne paraît possible que si chacun se voit comme ayant une identité culturelle suffisamment solide, c'est-àdire si chacun existe d'abord dans une relation positive avec ses propres normes et valeurs, davantage que par l'opposition ou dans une relation négative aux valeurs de l'autre. C'est par la connaissance ou la reconnaissance de sa propre culture et par la capacité de l'assumer que passe la possibilité de l'ouverture à d'autres cultures (Clanet 1990 : 220).

L'immigration est de plus en plus présente sur la scène muséale. On peut se demander si le Québec rejoindra cette vague de reconnaissance de l'histoire du passé et de celle du présent de l'immigration sur la place publique et muséale? 


\section{Références}

Arpin, Roland, 1998, Le Musée de la civilisation : une histoire d'amour. Québec, Musée de la civilisation et Fides.

Bergeron, Yves, 1996, Trésors d'Amérique française. Québec, Musée de l'Amérique française et Fides.

Clanet, Claude, 1990, L'interculturel. Introduction aux approches interculturelles en Éducation et en Sciences Humaines. Mirail, Presses universitaires du Mirail.

Clifford, James, 1997, Routes: Travel and Translation in the Late Twentieth Century. Cambridge (MA), Harvard University Press.

Curien, Pauline, 2003, "L'identité nationale exposée. Représentations du Québec à l'Exposition universelle de Montréal 1967 (Expo 67)». Thèse de doctorat en science politique, Faculté des sciences sociales, Université Laval.

Dubé, Philippe, 1991, «La mise en espace d'une ethnologie profonde: Mémoires au Musée de la civilisation». Ethnologie 14 (4): 31-38.

Gendreau, Andrée, 2003, La collection du Musée de la civilisation: principes et orientations. Québec, Musée de la civilisation.

—_ 2004, «Le Musée de la Civilisation et l'immigration : quel regard poser sur l'immigration? » Dans Musée et histoire de l'immigration, un enjeu pour toutes les nations, Colloque international, 9 et 10 décembre 2004, Paris, Bibliothèque nationale de France.

Létourneau, Jocelyn, 2004, Le Québec, les Québécois. Un parcours historique. Québec, Musée de la civilisation et Fides.

Loi sur les musées nationaux, s.d., Lois et règlements du Québec, chapitre M-44/5 (24.1), [En ligne] http://www.canlii.org/qc/legis/loi/m-44/ 20050111/tout.html

Mathieu, Jacques et Jacques Lacoursière, 1991, Les mémoires québécoises. Sainte-Foy, Les Presses de l'Université Laval.

Ministère de la Culture et des Communications, 2004, Créer comme personne d'autre! La diversité culturelle, une richesse pour le monde. Québec, Direction générale des affaires internationales et de la diversité culturelle.

Ministère des relations avec les citoyens et de l'immigration, mai 2004, Planification triennale de l'immigration 2005-2007. [En ligne] http:// www.micc.gouv.qc.ca/publications/pdf/Immigration_ Quebec_1999-2003.pdf. 
- 2005 , «Immigration au Québec selon les principaux pays de dernière résidence, $3^{\mathrm{e}}$ trimestre et 9 premiers mois de 2004 ». Bulletin statistique trimestriel sur l'immigration permanente au Québec. Québec, Direction de la population et de la recherche: 5 .

Musée de la civilisation, Québec, 2005a, Mémoires. [En ligne] http:// www.mcq.org/fr/mcq/memoires.html

Musée de la civilisation, Québec, 2005b, Le Temps des Québécois. [En ligne] http://www.mcq.org/fr/mcq/temps_quebecois.html

Musées et millénaire, $1^{\mathrm{er}}$ mars 2005, Identités américaines, [En ligne] http:/ /www.amerimumi.org/fr/index.htm

Pâquet, Martin, 1997, Vers un ministère québécois de l'immigration $1945-$ 1968. Ottawa, Société historique du Canada, coll. Les groupes ethniques du Canada 23.

— 2005, Tracer les marges de la cité. Étranger, immigrant et État au Québec, 1627-1981. Montréal, Boréal.

Sandell, Richard, 2002, "Museums and the combating of social inequality: roles, responsibilities, resistance ». Dans Richard Sandelle (dir.), Museums, Society, Inequality, Londres et New York, Routledge : 3-23.

Sauvage, Cécile, 2004, Mémoires au Musée de la civilisation de Québec. Regards croisés sur une exposition permanente. Québec, Les cahiers du Musée de la civilisation.

Taylor, Charles, 1992, Multiculturalisme, différence et démocratie. Paris, Flammarion. 
